\title{
Dissolution of amorphous silicon into solid aluminum
}

\author{
R. A. Scranton and J. O. McCaldin \\ California Institute of Technology, Pasadena, California 91125
}

(Received 24 January 1978; accepted 9 February 1978)

The rate of dissolution of amorphous $\mathrm{Si}$ into solid $\mathrm{Al}$ is measured at temperatures below $400^{\circ} \mathrm{C}$. The dissolution rate is found to be much faster than predicted by a simple model of the transport of $\mathrm{Si}$ through $\mathrm{Al}$. This result is related to defects in the growth of epitaxial $\mathrm{Si}$ using the solid-phase epitaxy process.

PACS numbers: $81.40 . \mathrm{Gh}, 63.0-. \mathrm{h}, 68.55 .+\mathrm{b}$

\section{INTRODUCTION}

Metal films are known to dissolve crystalline semiconductors at temperatures well below the eutectic temperature. Dissolution and transport of common semiconductors in contact with Au films have been demonstrated at room temperature. ${ }^{1}$ The solid Al metallization used in electronic devices is known to dissolve troublesome quantities of crystalline $\mathrm{Si}$ after heat treatment at $400^{\circ} \mathrm{C} .{ }^{2}$

Metal films are also known to dissolve amorphous semiconductors. Because of the high free energy associated with disorder, amorphous semiconductors are expected to have a higher driving energy for dissolution than crystalline semiconductors. The present investigation examines the dissolution at the amorphous $\mathrm{Si}$-solid-Al interface. The results are then related to problems in solid-phase epitaxy (SPE).

\section{SAMPLE PREPARATION AND MEASUREMENT}

The dissolution rate of amorphous $\mathrm{Si}$ into $\mathrm{Al}$ was measured by electron microprobe analysis after heat treatment at various temperatures (see Fig. 1).

Samples were prepared as follows. Single-crystal, polished sapphire substrates were cleaned in organics, a standard $\mathrm{Si}$ etch, a standard hydrogen peroxide based cleaning solution, rinsed in deionized water and dried in an oxygen atmosphere for one hour at $1000^{\circ} \mathrm{C}$. The substrates were loaded into an oil-free vacuum system. After reaching a pressure of $2 \times 10^{-8}$ Torr $\left(2.7 \times 10^{-6} \mathrm{~Pa}\right)$, separately shielded $\mathrm{Al}$ and Si sources were premelted. The substrates, on a heavy Cu block on a rotating shutter, were moved first into the flux of evaporating Si. The $a$-Si film thickness was monitored with a quartz crystal microbalance. Within seconds after finishing the Si evaporation, the samples were moved into the flux of evaporating Al. The Al film thickness was measured by a Nomarski interferometer after removal from the vacuum system. A typical structure was $\mathrm{Al}(10 \mu \mathrm{m}) / a$-Si $(450 \AA) / x-\mathrm{Al}_{2} \mathrm{O}_{3}$.

The vacuum system used in this work is very similar to those used in many of the reported SPE experiments. ${ }^{3-6}$ Electronbeam evaporation of $\mathrm{Si}$ in an ion-pumped system at pressures of approximately $10^{-7}$ Torr is quite standard. Roth and Anderson ${ }^{7}$ have noted several improvements in the SPE of the system $a-\mathrm{Si} / \mathrm{Pd} / x-\mathrm{Si}$ by using an ultrahigh vacuum of $10^{-9}$ to $10^{-10}$ Torr. The emphasis in this investigation was on more standard SPE conditions and hence a conventional highvacuum system was used.

Samples were quickly brought to heat treatment temperature by dropping them individually onto a heavy Cu block in an $\mathrm{Ar}$ atmosphere. To insure good thermal contact to the back of the sapphire, the surface of the Cu block was polished and wetted with liquid In. The temperature was stabilized to within $\mathrm{I}^{\circ} \mathrm{C}$ with a thermocouple and a proportional controller. The temperature was monitored with a digital thermometer with $0.2^{\circ} \mathrm{C}$ resolution, calibrated at the melting points of In, $\mathrm{Sn}$, and $\mathrm{Zn}$.

After heat treatment, a $\mathrm{Cu}$ vacuum chuck was used to quench the samples. Visual examination indicated that the In (melting point $156^{\circ} \mathrm{C}$ ) that adhered to the back of the substrate solidified as soon as the substrate flew from the heated block to the vacuum chuck (see Fig. 2). This procedure was timed by stopwatch to an accuracy of $\pm 0.2 \mathrm{~s}$. Within 1 $s$, the substrates were then placed into cold water to ensure complete cooling to room temperature.

After quench, the $\mathrm{Al}$ film and any $\mathrm{Si}$ in the $\mathrm{Al}$ film were removed by etching for $3 \mathrm{~h}$ in an etch containing phosphoric acid. This etch did not attack $a$-Si, as evidenced by the fact that a 200 - $\AA$-thick $a$-Si film was not etched in $3 \times 10^{3} \mathrm{~h}$.

Heated and unheated control samples were then examined in an optical microscope, scanning electron microscope, and an electron microprobe. Three different heat treat regimes were noted. For high temperatures, e.g. $525^{\circ} \mathrm{C}(10 \mathrm{~s})$, the 450- $\AA a$-Si films were completely dissolved. At medium temperatures, such as $400^{\circ} \mathrm{C}(10 \mathrm{~s})$, the $a$-Si film was covered by a layer of irregular Si precipitates of $\sim 1 \mu \mathrm{m}$ size. At low temperatures, such as $250^{\circ}-350^{\circ} \mathrm{C}(2.5-10 \mathrm{~s})$, the films were characterized by a uniformly thinned $a$-Si field with occasional isolated Si precipitates. The thickness of the uniform field in these specimens was measured by comparing the strength of the Si $k \alpha$ x-ray fluorescence in the electron microprobe with the strength of the signal from the unheated control. Uniformity of the field was verified by SEM and electron microprobe measurements. Typical results are indicated in Fig. 3 for the specimens in the low temperature regime.

\section{DISCUSSION}

The removal of $a$-Si by the $\mathrm{Al}$ is the result of two processes in series: the dissolution of the $a-\mathrm{Si}$ into the $\mathrm{Al}$ and the subse- 


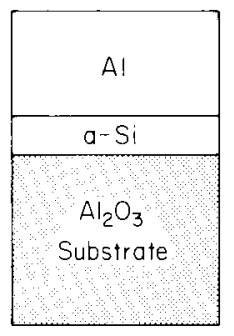

As Deposited

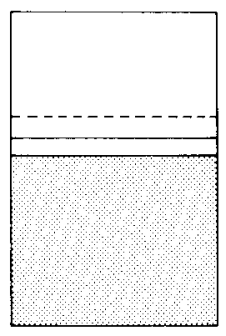

Heat Treated

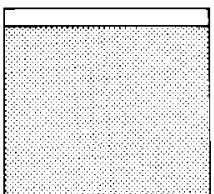

Etched
FIG. 1. The process used to measure of the dissolution rate of amorphous $\mathrm{Si}$ into solid $\mathrm{Al}$.

quent transport of Si away from the interface. The experimentally measured quantity, the rate of $a$-Si removal, is the smaller of the dissolution rate and the transport rate. Transport has been characterized as simple diffusion; the transport rate varies as $t^{-1 / 2}$, where $t$ is time. In contrast, the dissolution rate is constant in a simple model. Thus, for small $t$, the transport rate will exceed the dissolution rate. The thickness of $a$-Si removed should be limited by the dissolution process and hence linear in $t$ (see Fig. 4).

The experimental results were qualitatively in agreement with the above model; the thickness of the $a$-Si remaining on the substrate decreased linearly with $t$. Quantitatively, however, the above model fails.

The dissolution rate of the $a$-Si film into solid Al far exceeds the transport limit of the model. For example, the thickness of the $a$-Si film that dissolves in the $\mathrm{Al}$ in a transport (diffusion limited) model is

$$
Q=2 \sigma \sqrt{D t / \pi}
$$

where $Q$ is the thickness of $a$-Si removed, $\sigma$ is the solubility of $\mathrm{Si}$ in $\mathrm{Al}$ and $D$ is the diffusivity of $\mathrm{Si}$ in Al. Using values of $\sigma$ and $D$ measured by McCaldin and Sankur, ${ }^{4} Q$ for the $325^{\circ}$ $\mathrm{C}, 10$ s heat treat is $9 \AA$ A. In contrast, the electron microprobe indicates that $110 \AA$ has actually been removed. The small quantity of $\mathrm{Si}$ in the precipitates at the $\boldsymbol{a}$-Si surface does not account for the missing $\mathrm{Si}$.

The model must be modified to account for the rapid rate of removal of the $a$-Si by the solid Al. Al films saturated with $\mathrm{Si}$ are often found to have Si precipitates both within the Al and on nearby surfaces. ${ }^{8,9}$ Nucleation centers within a distance much less than $\sqrt{D t}$ of the interface act as sinks for the removal of Si from the Al film. In this way, the rate of removal of the $a$-Si could be very large, far exceeding the rate predicted by a transport limited model without precipitates. If the growing precipitates are very close to the $a$-Si film, the quantity of $a$-Si removed would be linear in time. ${ }^{10}$

The present model is thus one of a nutrient amorphous $\mathrm{Si}$ film, with a high free energy, feeding a number of precipitates, with only a small quantity of Si dissolved in the Al. The precipitates are in the Al film as well as on the $a$-Si surface. The precipitates are a more ordered phase of Si than the amorphous film, and hence there is a net driving force for transport from the amorphous film to the precipitates.

An important finding regarding the $a$-Si dissolution rate is the poor reproduceability of the experiment. Although all the evaporations were carried out in the same vacuum system, by the same investigator, using virtually identical procedures, the measured dissolution rates varied by an order of magnitude between samples prepared in separate evaporations (see Fig. 5). Samples prepared simultaneously in the vacuum system, but heat-treated separately always showed an increase in quantity of $a$-Si removed with an increase in either time or temperature of heat treatment. Samples prepared simultaneously gave identical dissolution rates when heated to the same temperature, suggesting that small differences in the quality of the vacuum, the evaporation rate, or the substrate temperature were responsible for the variation in dissolution rate. These variations could affect the nucleation sites and the type of disorder in the $\mathbf{a}-\mathrm{Si}$ as well as in the $\mathrm{Al}$.

\section{SOLID-PHASE EPITAXY.}

Solid-phase epitaxy refers to an epitaxial growth technique which utilizes thin solid films. Growth is driven by the lower free energy of the growing crystalline layer relative to an amorphous source layer. The SPE process is of potential interest to semiconductor device technology because of the low temperatures often employed $\left(300^{\circ}-600^{\circ} \mathrm{C}\right)$ compared to more conventional epitaxial growth processes $\left(900^{\circ}-1200^{\circ} \mathrm{C}\right)$. Low temperatures are attractive because the diffusivities of common dopants and impurities are reduced, resulting in more abrupt interfaces. In addition, the equilibrium concentration of defects is reduced at low temperatures.
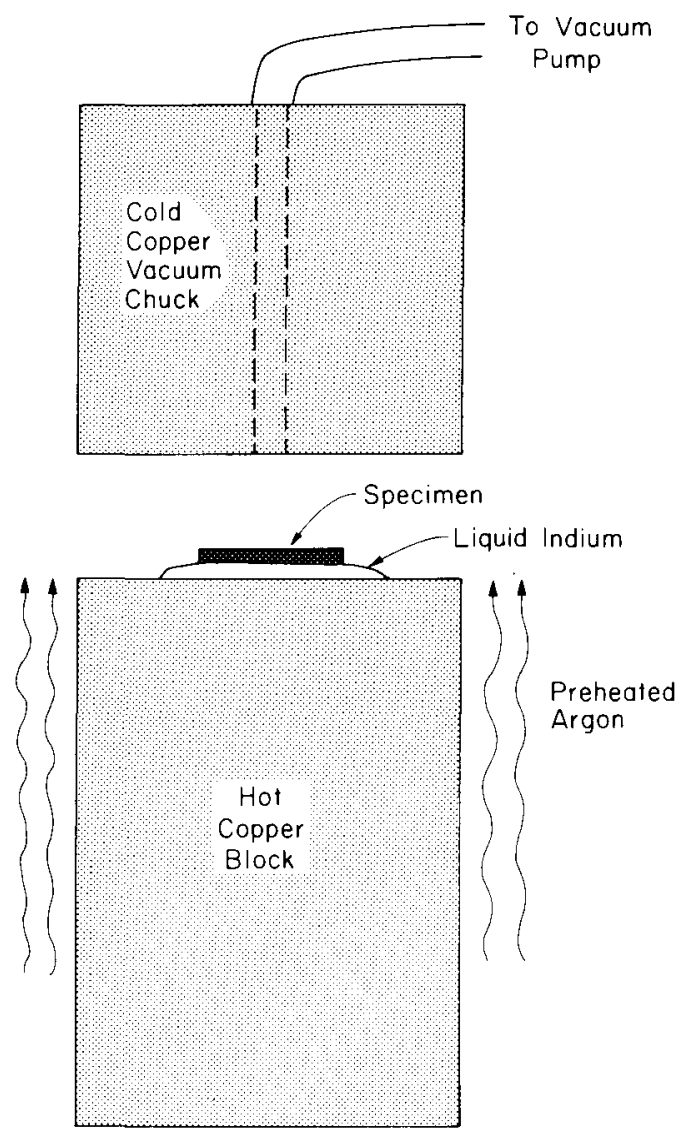

FIG. 2. Apparatus to heat treat specimens for short times. 


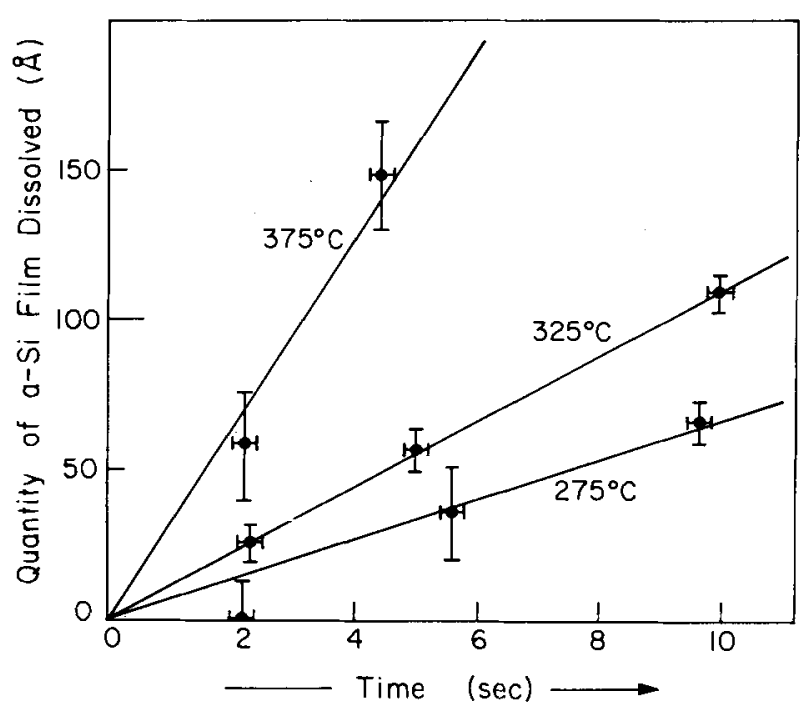

FIG. 3. The thickness of amorphous $\mathrm{Si}$ film removed by solid $\mathrm{Al}$ is shown as a function of time, with temperature as a parameter. Note the linear dependence on time.

An important SPE growth system that has attracted interest recently is a structure consisting of an amorphous Si film on an $\mathrm{Al}$ layer on a crystalline $\mathrm{Si}$ substrate $(a-\mathrm{Si} / \mathrm{Al} / x-\mathrm{Si})$. At temperatures below the $\mathrm{Si}-\mathrm{Al}$ eutectic $\left(577^{\circ} \mathrm{C}\right)$, the $a$-Si has been shown to dissolve into the $\mathrm{Al} \mathrm{film}$, transport through the $\mathrm{Al}$, and grow epitaxially on the substrate. The diffusivity of
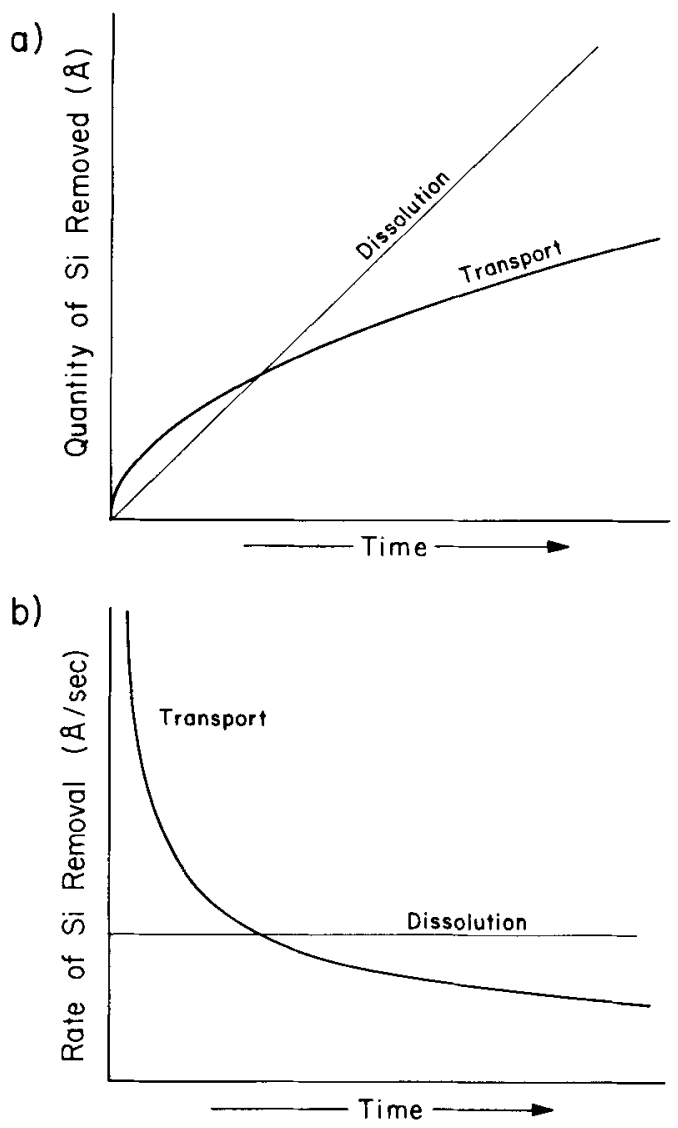

Fig. 4. (a) The thickness of amorphous silicon film removed as a function of time can be limited by either the dissolution process or the transport process. The corresponding rate of Si removal, shown in (b), will always be dissolution rate limited for short times.
$\mathrm{Si}$ in thin film $\mathrm{Al}$ has been shown to be enhanced by about one and one-half orders of magnitude over the diffusivity in bulk $\mathrm{Al}^{4}{ }^{4}$ However, the diffusivity in solids is still some three to five orders of magnitude smaller than the diffusivity in liquids and gases used in more conventional epitaxial growth. Nonetheless, SPE growth can still be very fast because the characteristic transport distance in $\mathrm{SPE}\left(10^{-5}-10^{-4} \mathrm{~cm}\right)$ is small compared to distances in more conventional epitaxial growth.

Indeed, the growth rate in SPE may be too fast. Fast growth rates can bury defects, such as metallic inclusions, in the epitaxial film. For example, Boatright and $\mathrm{McCaldin}^{5}$ have shown that transport-limited growth in SPE can cause pockets of $\mathrm{Al}$ to form in the epitaxial $\mathrm{Si}$ layer. These defects were found after heat treatment at $475^{\circ}-525^{\circ} \mathrm{C}$ for $10-20 \mathrm{~min}$. Majni and Ottaviani ${ }^{6}$ have demonstrated large-area, uniform growth of Si using SPE by heat treating an $a-\mathrm{Si} / \mathrm{Al} / x-\mathrm{Si}$ structure for $10 \mathrm{~h}$ at $450^{\circ}-530^{\circ} \mathrm{C}$. Such long times cause significant intermixing of the Si and Al films. Nevertheless, the epitaxial films prepared by Majni and Ottaviani also contained $\mathrm{Al}$ inclusions.

In more conventional epitaxy systems, the growth rate is controlled to prevent defects from being buried within the epitaxial layer. Control of the growth rate is often effected by control of the degree of saturation of the nutrient in the growth medium. In the isothermal $a-\mathrm{Si} / \mathrm{Al} / x$-Si SPE process, the corresponding variable is the rate of supply of the dissolved nutrient $\mathrm{Si}$ to the growing epitaxial region. This supply rate is the smaller of the rates of two series processes: the dissolution of $a-\mathrm{Si}$ into $\mathrm{Al}$ and the transport of Si through the $\mathrm{Al}$.

The present investigation, which demonstrates the poorly controlled, but rapid rate of dissolution, highlights a problem with the typical SPE technique: a lack of control of the growth rate. There is no simple dependence of the growth rate on an accessible experimental variable. For example, in the case of long heat treatment times used by Majni and Ottaviani, the $a$-Si is likely to have been converted to a more ordered phase,

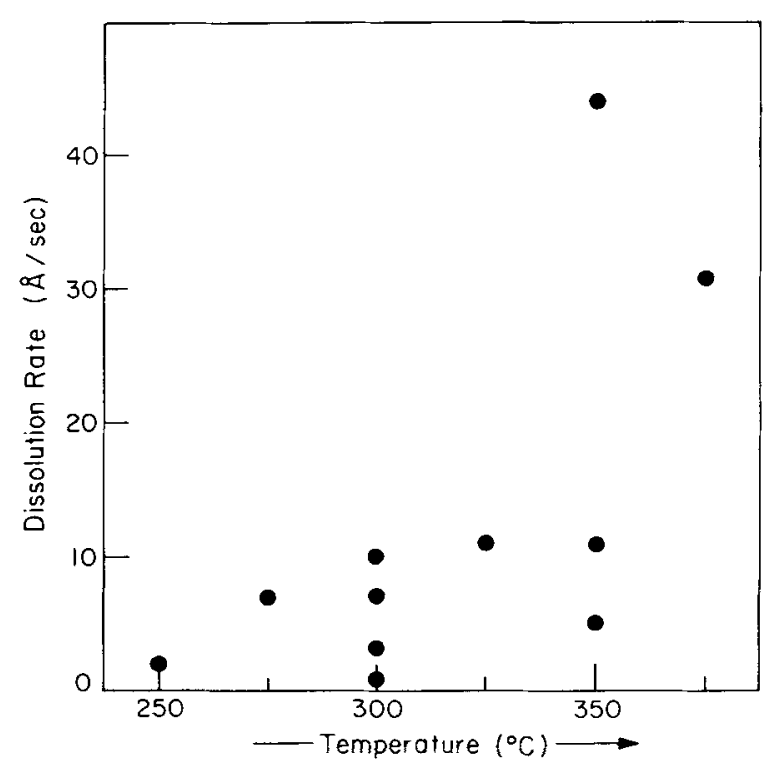

FIG. 5. The dissolution rate of amorphous $\mathrm{Si}$ into solid $\mathrm{Al}$ is plotted as a function of temperature for five separate evaporations. 
with the later stages of transport occurring from low energy precipitates and growths to the very low energy crystalline film. However, as a result of the initial high dissolution rate of the $a$-Si in close proximity to the substrate, defects are likely to have been buried.

A thin-film epitaxy process that does give control of the growth rate uses very thick metal films and a thickness of amorphous semiconductor to just saturate the metal at a particular heat treatment temperature. Initial heating would dissolve the amorphous semiconductor before significant growth has taken place. Growth could then be controlled by cooling the sample, as in liquid-phase epitaxy. For the $a$ $\mathrm{Si} / \mathrm{Al} / x$-Si SPE system, the epitaxial layer could be $1000 \AA$ thick for every $10 \mu \mathrm{m}$ of Al film thickness.

Control of the growth in Si/Al SPE can also suffer from the presence of impurities. When molecular-beam epitaxy (MBE) of $\mathrm{Si}$ is attempted at the $10^{-7}-10^{-6}$ Torr vacuum pressures often present during evaporation in Si SPE, the growths are of poor quality. ${ }^{11}$ Initial stages of growth in poor-vacuum $\mathrm{Si}$ MBE are characterized as three-dimensional, in contrast to the two dimensional growth of successful ultrahigh vacuum (UHV) Si MBE. Although the presence of metal in Si/Al SPE helps to clean the substrate, most Si SPE can still be characterized as three dimensional (island) growths. Thus, as presently constituted, Si SPE will probably require an UHV system. However, thin-film epitaxy of other semiconductors may not be so sensitive to impurities.

\section{v. CONCLUSIONS.}

The dissolution rate of $a$-Si into an evaporated $\mathrm{Al}$ film has been measured at temperatures below $400^{\circ} \mathrm{C}$ and shown to far exceed the transport rate of $\mathrm{Si}$ through the $\mathrm{Al}$ film. $\mathrm{Nu}$ cleation centers within the $\mathrm{Al}$ film are believed to remove $\mathrm{Si}$ from solution in the $\mathrm{Al}$ and cause the rapid decrease in the $a$-Si film thickness. Both the rapid dissolution and the lack of control of the dissolution are related to problems of $\mathrm{Si} / \mathrm{Al}$ SPE.

\section{ACKNOWLEDGMENTS}

The authors thank J. S. Best for his assistance with the heat treatments. Work was supported in part by the Office of Naval Research (L. Cooper) and the Army Research Office (H. Wittman).

\footnotetext{
${ }^{1}$ A. Hiraki, K. Shuto, S. Kim, W. Kammura, and M. Iwami, Appl. Phys. Lett. 31, 611 (1977).

${ }^{2}$ C. T. Kircher, J. Appl. Phys. 47, 5394 (1976).

${ }^{3}$ K. Nakamura, M-A. Nicolet, J. W. Mayer, R. J. Blattner, and C. A. Evans, Jr., J. Appl. Phys. 46, 4678 (1975).

${ }^{4}$ J. O. McCaldin and H. Sankur, Appl. Phys. Lett. 19, 524 (1971).

${ }_{5}^{5}$. L. Boatright and J. O. McCaldin, J. Appl. Phys. 47, 2260 (1976).

${ }^{6}$ G. Majni and G. Ottaviani, Appl. Phys. Lett. 31, 126 (1977).

${ }^{7}$ John A. Roth and C. Lawrence Anderson Appl. Phys. Lett. 31, 689 (1977).

${ }^{8}$ G. J. van Gurp, J. Appl. Phys. 44, 2040 (1973).

9J. O. McCaldin and H. Sankur, Appl. Phys. Lett. 20, 171 (1972).

${ }^{10}$ Strictly, if the nucleation centers (sinks for the $\mathrm{Si}$ ) are all located a distance $h$ away from the interface, then the measured quantity of $a$-Si removed will be linear in $t$ for times long compared to $h^{2} / D$. The measured rate would then be the lower of the dissolution rate or the transport-limited rate given by $\sigma D / h$. Thus the measured rate is a lower bound for the dissolution rate.

"Yusuke Ota, J. Electrochem. Soc. 124, 1795 (1977).
} 